\title{
Qualité de la membrane des spermatozoïdes de truite arc-en-ciel (Oncorhynchus mykiss); relation avec l'aptitude du sperme à la congélation
}

\author{
Marie-Laure Malejac, Maurice Loir \\ et Gérard Maisse
}

INRA, Laboratoire de Physiologie des Poissons, Campus de Beaulieu, 35042 Rennes Cedex, France. Reçu le i 3 juillet i 989 , accepté le 28 septembre i 989 .

Membrane properties of spermatozoa from rainbow trout (Oncorhynchus mykiss) and their relationship to the freezing ability of sperm.

Malejac M.-L., M. Loir, G. Maisse. Aquat. Living Resour., 1990, 3, 43-54.

Abstract

Résumé
Milt was collected from two groups of 20 males each, once a week for 10 weeks from springspawning rainbow trout strain (Oncorhynchus mykiss). Only semen with a volume greater than $5 \mathrm{~cm}^{3}$ were studied. Physico-chemicals characteristics of different milts were measured (spermatozoa concentration, osmolality, glutamic oxaloacetic transaminase (GOT) and $17 \alpha, 20 \beta$-dihydroxy-4-pregnen-3-one $(17 \alpha, 20 \beta-\mathrm{OH}-\mathrm{P})$ levels in the seminal fluid). Spermatozoa samples were submitted to swelling tests in hypotonic solutions (60 and $20 \mathrm{mOsm})$ over 1 and 24 hours and their response monitored (swelling, bursting and stainability with propidium iodide). A sample of each milt was frozen into pellets and the quantity of GOT released by the spermatozoa was measured after thawing. Fertilization tests were done with both fresh and cryopreserved sperm.

Factorial analysis of correspondences was performed between fresh and frozen milt fertility versus physico-chemical characteristics and membrane quality. Fitness for cryopreservation can be established a priori by measuring seminal osmolality and performing tests which indicate the membrane's functional integrity. These results confirm the membrane determining role during freezing.

Keywords : Trout, sperm, cryopreservation, membrane quality.

Les spermes de deux lots de 20 mâles d'une souche de truite arc-en-ciel (Oncorhynchus mykiss) à reproduction printanière, ont été collectés individuellement une fois par semaine pendant 10 semaines. Seuls les spermes d'un volume supérieur à $5 \mathrm{~cm}^{3}$ ont été utilisés pour cette étude. Pour chacun, la concentration en spermatozoïdes, l'osmolarité et les concentrations enzymatiques en glutamique oxaloacétique transaminase (GOT) et stérö̈dienne en $17 \alpha, 20 \beta$-dihydroxy-4-pregnen-3-one (17 $\alpha, 20 \beta$ $\mathrm{OH}-\mathrm{P})$ du plasma séminal ont été mesurées. Des échantillons de spermatozoïdes ont été incubés pendant 1 et 24 heures dans des solutions hypotoniques (60 et $20 \mathrm{mOsm}$ ) et leurs réactions (gonflement, éclatement, colorabilité ou non par l'iodure de propidium) ont été analysées. Un échantillon de chaque sperme a été congelé en boulettes à partir desquelles la GOT libérée par les spermatozoïdes après décongélation a été dosée. Des fécondations ont été réalisées avec du sperme frais et du sperme congelé-décongelé.

L'analyse factorielle des correspondances (AFC) entre la fécondance du sperme après congélation d'une part et la fécondance à l'état frais, les caractéristiques physico-chimiques et les résultats des différents tests de qualité membranaire d'autre part, a montré que l'aptitude à la cryoconservation peut être estimée a priori à partir de la mesure de la pression osmotique séminale et de tests reflétant l'intégrité fonctionnelle de la membrane. Ce résultat confirme le rôle déterminant de la membrane lors de la congélation.

Mots-clés : Truite, sperme, congélation, qualité de la membrane. 


\section{INTRODUCTION}

La congélation du sperme de salmonidés a fait l'objet de nombreuses recherches (revues bibliographiques de Scott et Baynes, 1980 et de Stoss, 1983) qui ont permis la mise au point d'une technique de congélation/décongélation, de dilueurs et de cryoprotecteurs appropriés. Il reste cependant un certain nombre de problèmes à résoudre. Notamment, une grande variabilité des résultats a pu être constatée (Stoss et Holtz, 1983; Maisse et al., 1988) entre les éjaculats d'un même mâle et au cours de la période de reproduction. Ce fait justifie la recherche d'un test qui permettrait de prévoir a priori la résistance à la congélation de chaque sperme et de congeler uniquement ceux qui se révéleraient les meilleurs.

Lors de la congélation/décongélation et malgré les précautions prises, la membrane est altérée (Yoo et al., 1987; Schmehl et al., 1987). Ceci se traduit entre autre par la perte par les spermatozoïdes de protéines que l'on retrouve alors dans le dilueur de congélation. Baynes et Scott (1982) ont suggéré que les altérations de la membrane pourraient avoir une influence directe sur la réussite de la fécondation après décongélation. Cette idée a été confortée par des travaux de Maisse et al. (1988) qui ont montré une relation inverse entre l'aptitude à la congélation et la concentration dans le plasma séminal d'une protéine d'un poids moléculaire de $42 \mathrm{KD}$, qui est l'un des constituants majeurs de la membrane des spermatozoïdes. Ces données nous ont conduit à nous intéresser plus précisément à la membrane des spermatozoïdes.

Dans cette étude, nous avons caractérisé chaque sperme, dont la fécondance a été testée après congélation, par des critères dont les variations au cours de la période de spermiation ont été décrites. Nous avons utilisé deux voies d'approches pour caractériser la membrane des spermatozoïdes : une approche descriptive sur le sperme frais et une approche expérimentale consistant à tester la résistance de la membrane par un choc hypoosmotique.

\section{MATÉRIELS ET MÉTHODES}

\section{Les mâles et la collecte du sperme}

Les 40 géniteurs mâles (âge : 2 ans) utilisés appartenaient à une souche de truite arc-en-ciel (Oncorhynchus mykiss) à reproduction printanière, en provenance de la pisciculture INRA de Gournay-surAronde. Après séparation en deux lots de 20 poissons, ils ont été marqués individuellement (marque de type « Flow Tag FD-68B » numérotée) puis transférés dans un circuit fermé thermorégulé $\left(12-13^{\circ} \mathrm{C}\right)$ le 25 mars 1988. A cette date, tous les mâles étaient déjà spermiants. Les poissons ont été maintenus à jeun pendant la durée de l'expérience. A chaque manipulation, les animaux étaient anesthésiés dans un bain de 2-phénoxyéthanol $\left(3 \mathrm{~cm}^{3} / 10 \mathrm{dm}^{3}\right.$ d'eau $)$ puis traités au vert malachite.

Une collecte hebdomadaire a été pratiquée sur chaque lot à l'aide d'une pipette de transfert à usage unique, qui permet l'aspiration de sperme non contaminé (eau, fécès, urines) dont l'émission est obtenue par pression des flancs de l'animal. En raison du nombre des analyses pratiquées, seuls les spermes dont le volume était supérieur à $5 \mathrm{~cm}^{3}$ ont été étudiés. Pendant les manipulations, le sperme frais était conservé sur de la glace fondante à l'abri de l'eau et de la lumière.

\section{La congélation du sperme}

La congélation du sperme a été effectuée rapidement après les prélèvements. Le sperme frais était tout d'abord centrifugé 20 minutes à $500 \mathrm{~g}$ à $4{ }^{\circ} \mathrm{C}$. Le culot de spermatozoïdes était dilué avec 3 volumes de dilueur de Mounib (1978) modifié (Sucrose : 125 mmol, gluthation réduit : $6,5 \mathrm{mmol}$, bicarbonate de potassium: $100 \mathrm{mmol}$, jaune d'œuf au tellurite de potassium : $8 \%$, diméthylsulfoxide (DMSO) : $8 \%$ ). La concentration de DMSO utilisée diffère de celle proposée par Legendre et Billard (1980) qui utilisent $10 \%$ de DMSO. Compte tenu des résultats de Erdahl (1986) montrant un effet toxique du DMSO apparaissant pour des concentrations supérieures ou égales à $10 \%$, nous avons préféré en diminuer la concentration. Le mélange a été congelé sous forme de boulettes de $0,1 \mathrm{~cm}^{3}$ selon la technique de Nagase (1964) et stocké dans de l'azote liquide.

\section{Fécondation à l'état frais et après congélation}

\section{Fécondation avec du sperme frais}

La fécondance de chaque sperme frais a été obtenue par fécondation, à la dilution $10^{-3}$ dans du DIA 532 (Billard, 1977), d'ovules ( $n=$ environ 200) provenant d'au moins deux femelles de la même souche que les mâles. Parallèlement, la fécondabilité des ovules a été évaluée à partir de la fécondation de deux lots de $15 \mathrm{~g}$ avec du sperme à la dilution $10^{-1}$. Les incubations se sont déroulées dans un circuit fermé thermorégulé à $10^{\circ} \mathrm{C}$. Le taux d'embryonnement à 22 jours a été déterminé par comptage direct des œufs œillés. La fécondance est exprimée par rapport à la fécondabilité des ovules. Le taux d'éclosion a été noté afin d'estimer le pourcentage d'embryons anormaux.

\section{Fécondation avec du sperme congelé}

Une boulette est placée à côté d'un mélange d'ovules (15 g; au moins deux femelles) dans un bol et simultanément, on verse délicatement $10 \mathrm{~cm}^{3}$ de « DIA 532 » maintenu à $25^{\circ} \mathrm{C}$. L'ensemble est rapidement homogénéisé par transvasements successifs d'un récipient dans l'autre. La suite de la manipulation est la même que pour l'insémination avec du sperme frais. Le délai écoulé entre la congélation et 
la fécondation a varié de 3 à 50 jours selon les échantillons. Comme pour le sperme frais, la fécondance est exprimée par rapport à la fécondabilité des ovules et le taux d'éclosion a été noté.

\section{Les caractéristiques physico-chimiques du sperme frais}

- Le nombre de spermatozoïdes par $\mathrm{cm}^{3}$ de sperme (calculé après dilution au 1/10000) a été déterminé à l'aide d'une cellule de Thomas.

- L'osmolarité du plasma séminal a été mesurée à l'aide d'un osmomètre cryoscopique.

- La concentration d'une enzyme intramitochondriale, la glutamique oxaloacétique transaminase ou GOT, a été mesurée dans le plasma séminal. La présence de cette enzyme dans le plasma séminal résulterait d'une altération de la membrane des spermatozoïdes (Erdahl, 1986; Schmehl et al., 1987). Le dosage est réalisé à l'aide d'un coffret de réactifs Boehringer (référence 258784). Les mesures $\left(\mathrm{mU} / 100 \mathrm{~mm}^{3}\right.$ de culot à $\left.500 \mathrm{~g}\right)$ ont été réalisées au spectrophotomètre à $340 \mathrm{~nm}$. La justesse du dosage a été vérifiée avec une solution de référence (Precinorm U, Boehringer). La variabilité du dosage, établie à l'aide de 14 mesures à partir d'un mélange de spermes, était de $\pm 5 \%$.

- La concentration de la $17 \alpha, 20 \beta$-dihydroxy-4pregnen-3-one (17 $\alpha, 20 \beta-\mathrm{OH}-\mathrm{P})$ dans le plasma sémnial a été déterminée par dosage radioimmunologique, effectué selon la méthode décrite par Fostier et Jalabert (1986) après extraction à l'acétate d'éthyle : cyclohexane $(50: 50)$.

\section{Tests de la qualité membranaire des spermatozoïdes}

L'aptitude de la membrane des spermatozoïdes à supporter un choc hypoosmotique a été estimée par :

$1^{\circ}$ La détermination du pourcentage de cellules dont la membrane est gonflée ou éclatée après incubation pendant 1 et 24 heures dans une solution à 60 mOsm (20 $\mathrm{cm}^{3}$ de milieu minéral du liquide séminal, ou MMLS, Billard et Jalabert (1974) à 285 mOsm étendus à $560 \mathrm{~cm}^{3}$ avec de l'eau distillée et auxquels $1,16 \mathrm{~g}$ de $\mathrm{KCl}$ était ajouté). Après une première dilution $\left(10^{-2}\right)$ dans la solution hypoosmotique et une incubation de 30 minutes à $12-13^{\circ} \mathrm{C}$, une nouvelle dilution $\left(10^{-2}\right)$ était réalisée soit juste après l'incubation (observation " 1 heure ») soit après 24 heures de stockage à $4{ }^{\circ} \mathrm{C}$ (observation « 24 heures »). Trois types de réponses ont été distingués ( fig.

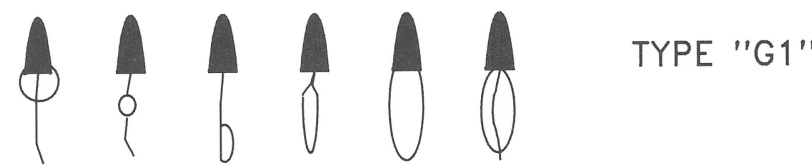

Dif 19

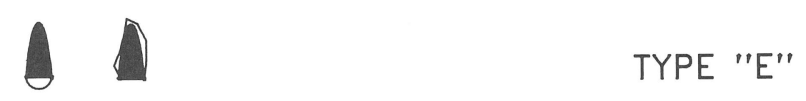

Figure 1. - Représentation schématique des différentes formes de gonflement observées au microscope optique en contraste de phase $(\mathrm{G} \times 200)$, à la dilution $10^{-4}$ avec : formes $\mathbf{G 1}$ : gonflement au niveau du flagelle du spermatozoïde; formes G2: gonflement au niveau de la tête et du flagelle du spermatozoïde, formes $\mathbf{E}$ : membrane éclatée.

Schematic representations of different swelling forms observed via optical microscope $(M \times 200)$, dilution $10^{-4}$. G1 form: swelling around flagellum: G2 form: swelling around head and flagellum; $\mathbf{E}$ form: broken membrane.

1) : le type G1 (gonflement au niveau du flagelle), le type G2 (gonflement au niveau du flagelle et de la tête du spermatozoïde) et le type $\mathbf{E}$ (membrane éclatée). Les comptages ont été réalisés à l'aide d'un hématimètre au microscope optique à contraste de phase $(\mathrm{G} \times 200)$. La technique a été validée par addition à du sperme frais de quantités croissantes de sperme chauffé (tableau 1).

$2^{\circ}$ La détermination du pourcentage de spermatozoïdes dont la membrane ne résiste pas à une incubation de 30 minutes dans une solution à $20 \mathrm{mOsm}$ $\left(3,6 \mathrm{~cm}^{3}\right.$ MMLS étaient étendus à $400 \mathrm{~cm}^{3}$ avec de l'eau distillée et auxquels 0,208 g de $\mathrm{KCl}$ était ajouté).

Après une incubation préalable de 30 minutes à $12{ }^{\circ} \mathrm{C}$ de sperme frais à la dilution $10^{-2}$ dans la solution à $20 \mathrm{mOsm}$, une fraction était reprise et diluée au $1 / 3$ avec cette solution puis incubée ( 3 minutes) après addition d'une solution mère d'iodure de propidium (PI) (1 mg de PI dans $50 \mathrm{~cm}^{3}$ de DPBS - Dulbecco's phosphate buffered saline) à raison de $60 \mathrm{~mm}^{3}$ pour $400 \mathrm{~mm}^{3}$ de suspension de sperme incubée. La viabilité des cellules (coloration en rouge des cellules mortes) a été déterminée à l'aide d'un microscope à fluorescence.

L'aptitude de la membrane à supporter la congélation-décongélation a été estimée à l'aide du dosage de la GOT libérée pendant cette étape dans le dilueur de congélation. Le dosage a été réalisé comme indiqué 
Tableau 1. - Évolution des pourcentages des formes de gonflement des spermatozoïdes de type $\mathbf{G 1}, \mathbf{G} \mathbf{2}$ et $\mathbf{E}$ en fonction de la proportion de spermatozoïdes chauffés $\left(56^{\circ} \mathrm{C}, 30\right.$ minutes).

Change in the percentages of spermatozoa swelling forms G1, G2 and $\mathbf{E}$, with increasing quantities of heated spermatozoa $\left(56^{\circ} \mathrm{C}\right.$, 30 minutes).

\begin{tabular}{ccrr}
\hline $\begin{array}{c}\text { Pourcentage de spermatozoïdes } \\
\text { chauffés dans une solution } \\
60 \text { mOsm }\end{array}$ & G1 & G2 & E \\
\hline 0 & 54 & 37 & 9 \\
20 & 45 & 32 & 23 \\
40 & 31 & 20 & 49 \\
60 & 20 & 12 & 68 \\
80 & 0 & 0 & 100 \\
\hline
\end{tabular}

plus haut. Chaque boulette de sperme congelé était déposée rapidement dans un tube contenant $2 \mathrm{~cm}^{3}$ de DIA 532 maintenu à $30^{\circ} \mathrm{C}$ dans un bain-marie à agitation. Le tube bouché était agité rapidement par retournements successifs et placé immédiatement après la fusion (la plus rapide possible afin de minimiser les altérations dues à la décongélation) sur un portoir à $12-13^{\circ} \mathrm{C}$. Après centrifugation $(500 \mathrm{~g}-20 \mathrm{mi}-$ nutes), le surnageant était récupéré et dosé pour connaitre la concentration de GOT dans le dilueur (en $\mathrm{mU} / 100 \mathrm{~mm}^{3}$ de culot à $500 \mathrm{~g}$ ).

L'activité enzymatique dosée dans les culots (repris par $2 \mathrm{~cm}^{3}$ de DIA, soumis aux ultrasons puis centrifugés à $1500 \mathrm{~g}$ pendant 20 minutes), additionnée à celle trouvée dans les surnageants a permis de connaître la quantité de GOT présente initialement dans les spermatozoïdes.

\section{Analyses statistiques}

Les corrélations entre la fécondance du sperme congelé et l'ensemble des autres paramètres pris en considération ont été étudiées après réalisation d'un tableau de contingence croisant les modalités de la variable "fécondance du sperme congelé 》 avec les modalités des variables "fécondance du sperme frais ", "caractéristiques physico-chimiques » et " tests de qualité membranaire des spermatozoïdes ». Les modalités ont été établies d'après l'étude des histogrammes de distribution de chaque variable. Nous avons ensuite procédé à une analyse factorielle des correspondances sur le tableau de contingence ainsi créé (Benzecri, 1980) à l'aide d'un logiciel STATITCF dont la programmation a été réalisée d'après Foucart (1984).

La signification des variations des différents paramètres au cours de la période d'étude a été analysée à l'aide du test U de Mann et Whitney.

\section{RÉSULTATS}

Les valeurs moyennes et extrêmes obtenues pour quelques paramètres des spermes étudiés sont réunies dans le tableau 2.

La fécondance du sperme frais varie selon les mâles et les lots d'ovules inséminés tout en révélant une

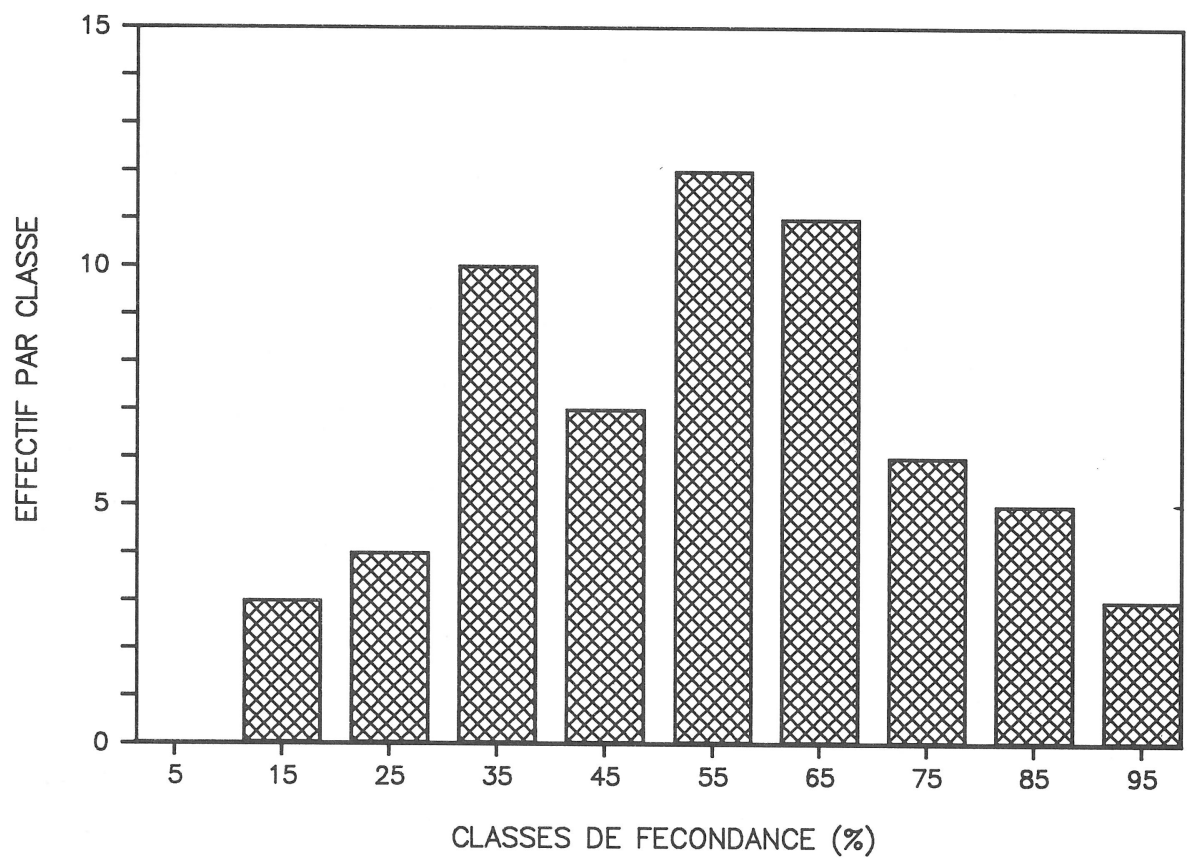

Figure 2. - Distribution des classes de fécondance après cryoconservation (nombre total des spermes: 61).

Distribution of fertility classes after cryopreservation (total sperm number: $61)$. 
Tableau 2. - Caractéristiques des spermes étudiés.

Studied sperms characteristics.

\begin{tabular}{|c|c|c|}
\hline Paramètre étudié & Valeur moyenne & Valeurs extrêmes \\
\hline $\begin{array}{l}\text { Concentration en spermato- } \\
\text { zoïdes }\left(n / \mathrm{cm}^{3}\right)\end{array}$ & $\begin{array}{l}2,05 \times 10^{10} \\
(n=65)\end{array}$ & $0,8-3,3 \times 10^{10}$ \\
\hline Pression osmotique (mOsm) & $\begin{array}{c}265 \\
(n=65)\end{array}$ & $180-320$ \\
\hline $17 \alpha, 20 \beta-\mathrm{OH}-\mathrm{P}\left(\mathrm{ng} / \mathrm{cm}^{3}\right)$ & $\begin{array}{c}4,41 \\
(n=64)\end{array}$ & $0,037-20,60$ \\
\hline $\begin{array}{l}\text { GOT plasma séminal frais } \\
\left(\mathrm{mU} / 100 \mathrm{~mm}^{3} \text { culot à }\right. \\
500 \mathrm{~g})\end{array}$ & $\begin{array}{c}1,94 \\
(n=61)\end{array}$ & $0,10-4,82$ \\
\hline $\begin{array}{l}\text { GOT totale }\left(\mathrm{mU} / 100 \mathrm{~mm}^{3}\right. \\
\text { culot à } 500 \mathrm{~g})\end{array}$ & $\begin{array}{c}345,4 \\
(n=61)\end{array}$ & $140-520$ \\
\hline $\begin{array}{l}\text { GOT Cryoconservateur } \\
\left(\mathrm{mU} / 100 \mathrm{~mm}^{3} \quad \text { culot à }\right. \\
500 \mathrm{~g})\end{array}$ & $\begin{array}{c}137,1 \\
(n=61)\end{array}$ & $70,8-256,5$ \\
\hline
\end{tabular}

homogénéité des résultats vers de forts pourcentages $(>70 \%$ ) avec une moyenne de 94,6\%. La fécondance du sperme congelé varie beaucoup, de 10 à $100 \%$ (fig. 2). Le taux d'éclosion a toujours été de $100 \%$.

Les 65 spermes testés ont été prélevés sur 34 mâles. Seuls six mâles ont permis un suivi individuel des différents paramètres pendant 6 semaines à raison de 4 à 5 prélèvements par poisson pour l'ensemble de la période considérée. L'observation de l'évolution des paramètres obtenus révèle de fortes variations intermâles et au cours de la saison de spermiation pour un même animal.

La fécondance du sperme après congélation varie de manière importante suivant les poissons et la date de collecte (variation de $72 \%$ entre le plus faible et le plus fort pourcentage pour un mâle donné). L'étude des moyennes par prélèvement montre des fluctuations significatives des fécondances pendant la période (fig. 3).

La concentration en spermatozoïdes varie de manière importante selon les poissons et la date de collecte (du simple au triple). L'étude des moyennes par prélèvement et par lot révèle une tendance à la diminution au cours de la période (fig. 4).

L'étude des plasmas séminaux montre des différences entre mâles et entre prélèvements : il en est ainsi de la pression osmotique pour laquelle les valeurs sont très dispersées de 180 à $320 \mathrm{mOsm}$, avec une moyenne de $265 \mathrm{mOsm}$. Les variations sont synchrones pour les deux lots, avec une baisse significative pendant la deuxième quinzaine d'avril (fig. 5). Les concentrations de $17 \alpha, 20 \beta-\mathrm{OH}-\mathrm{P}$ varient de 0,037 à $20,6 \mathrm{ng} / \mathrm{cm}^{3}$ et les évolutions selon les mâles peuvent être très différentes. L'évolution des moyennes confirme cette forte variation au cours de la période de prélèvement avec une diminution significative pour un des lots à partir de la mi-avril (fig. 6). La distribution des concentrations de la GOT dans le plasma séminal frais est très étroite (de 0,1 à $4,82 \mathrm{mU} / 100 \mathrm{~mm}^{3}$ de culot à $500 \mathrm{~g}$ ), les variations notées entre les spermes étant à la limite de la sensibilité du dosage lui-même.

La concentration en GOT totale est très élevée (140 à $520 \mathrm{mU} / 100 \mathrm{~mm}^{3}$ de culot à $500 \mathrm{~g}$ ) par rapport à celle présente dans le plasma séminal frais. Les variations intermâles ou pour un même mâle peuvent être très importantes, allant du simple au double. Cependant, l'observation de l'évolution des moyennes par

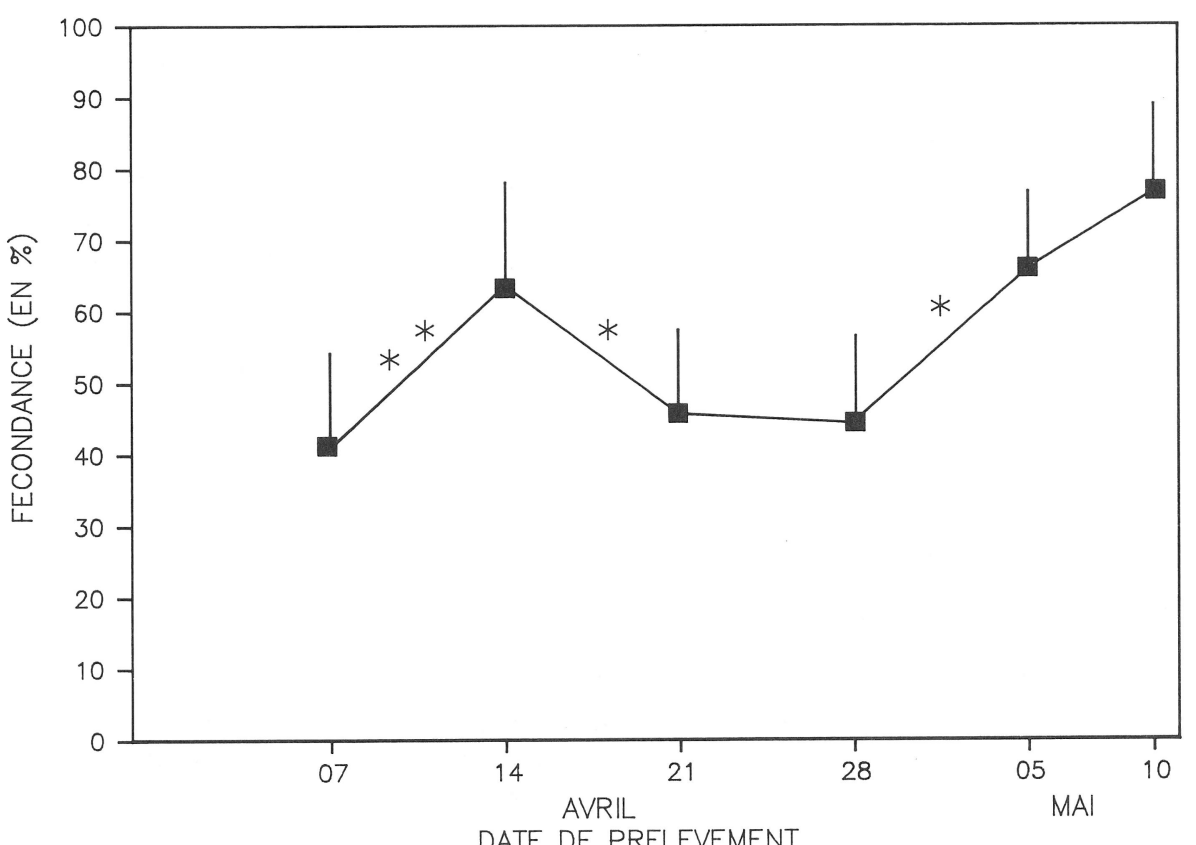

DATE DE PRELEVEMENT
Figure 3. - Évolution des moyennes des fécondances du sperme congelé pendant 6 semaines pour un lot de mâles (moyenne + écart type : $n=8$ à 10 spermes). Niveau de signification des différences observées $\left({ }^{*}\right) p \leqq 0,05$; $\left.{ }^{* *}\right) p \leqq 0,01$.

Change in frozen sperm fertility for one group (mean $+S E ; n=8$ to 10). Significance level of observed differences $\left(^{*}\right) p \leqq 0.05 ;\left(^{* *}\right) p \leqq 0.01$. 


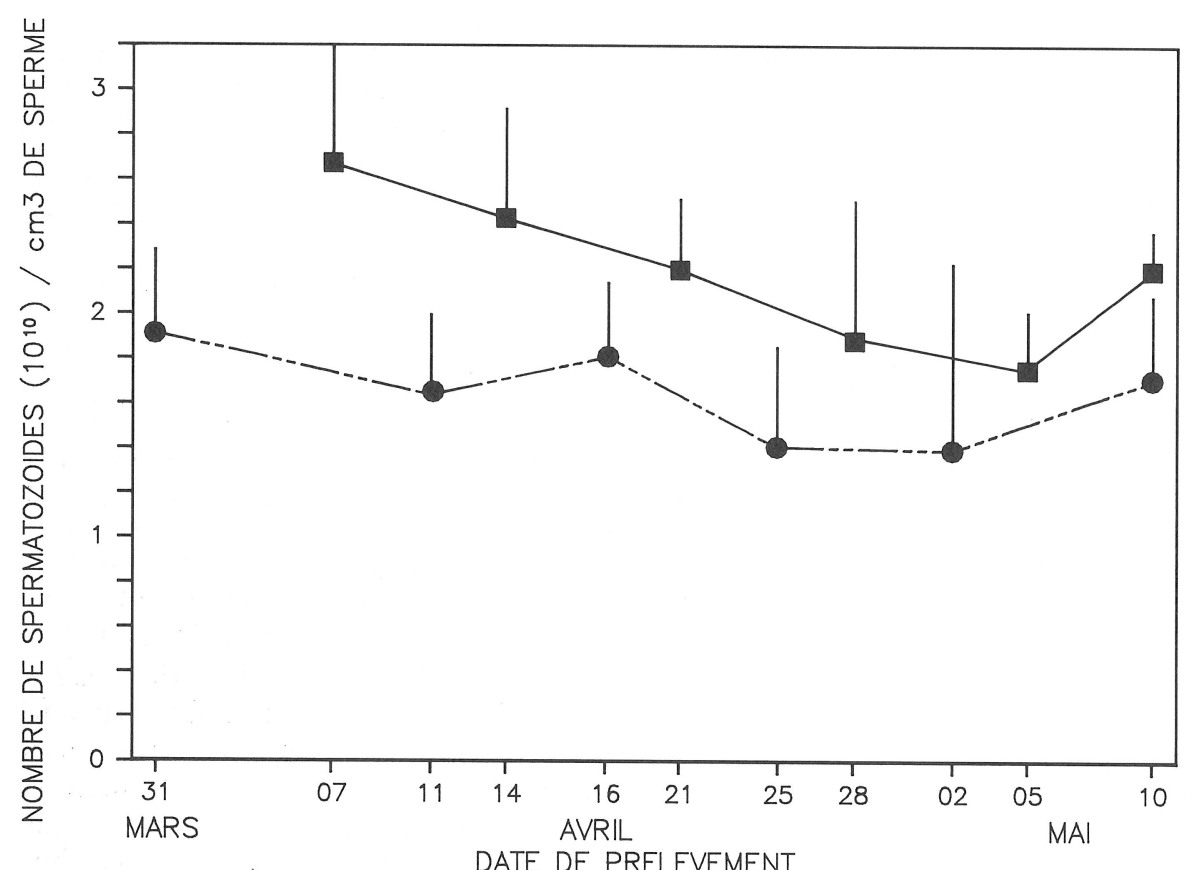

DATE DE PRELEVEMENT

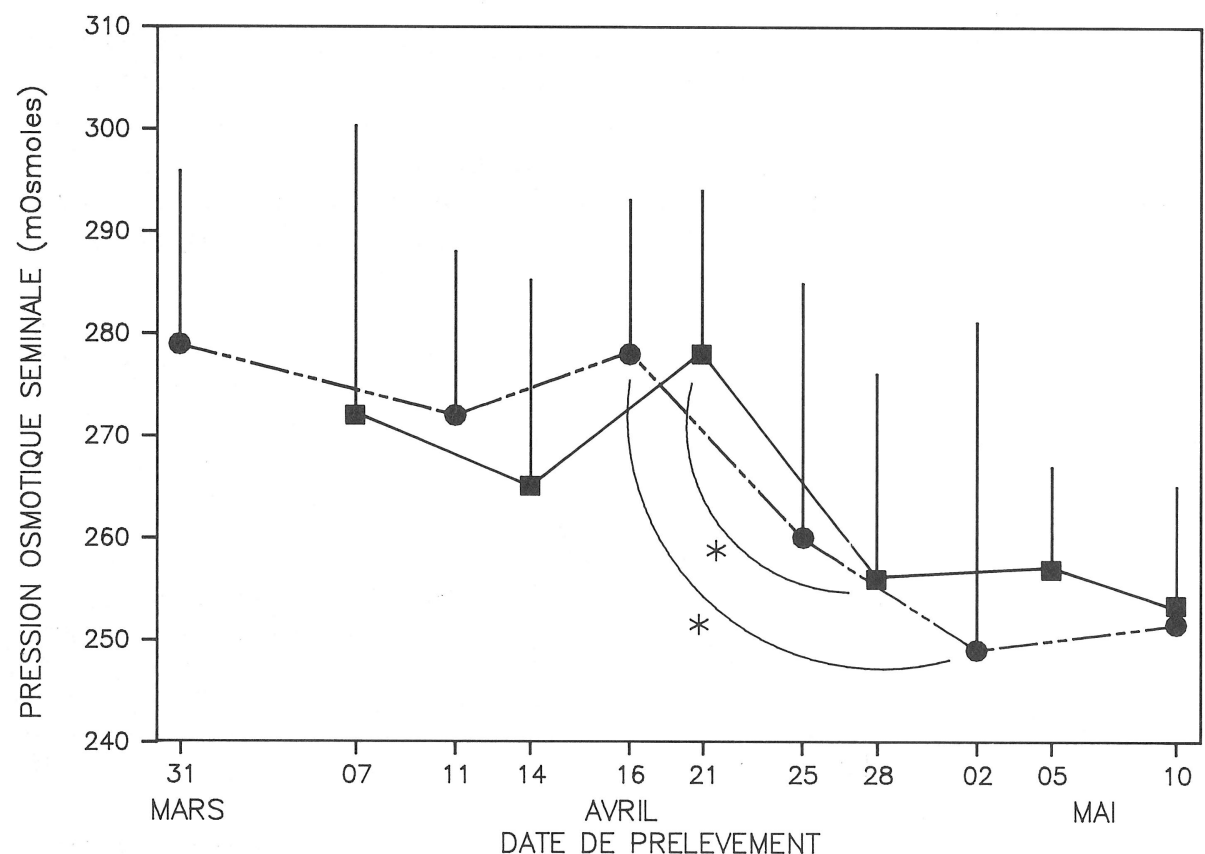

Figure 4. - Évolution des moyennes du nombre de spermatozoïdes par $\mathrm{cm}^{3}$ de sperme $\left(\times 10^{10}\right)$ pour les deux lots de mâles (moyenne +écart type; $n=8$ à 10 spermes). Niveau de signification des différences observées $(*)$ $p \leqq 0,05 ;(* *) p \leqq 0.01$.

Change in number of spermatozoa per $\mathrm{cm}^{3}\left(\times 10^{10}\right)$ for the two male groups (mean $+S E ; n=8$ to 10). Significance level of observed difference (*) $p \leqq 0.05 ;(* *) p \leqq 0.01$.

Figure 5. - Évolution des moyennes des pressions osmotiques séminales (mOsm) pour les deux lots de mâles (moyenne+écart type; $n=8$ à 10 spermes). Niveau de signification des différences observées $\left.{ }^{*}\right) p \leqq 0,05$; **) $p \leqq 0,01$.

Change in osmolality ( $\mathrm{mOsm}$ ) for the two male group (mean $+S E ; n=8$ to 10). Significance level of observed differences $\left(^{*}\right) p \leqq 0.05 ;\left({ }^{* *}\right) p \leqq 0.01$. lot sur l'ensemble de la période de prélèvement montre une relative constance dans les résultats, sans révéler de variations entre le début et la fin de la période des manipulations (fig. 7).

L'étude des pourcentages de spermatozoïdes de type $\mathbf{E}$ à 1 heure montre que les variations sont importantes selon les mâles considérés, les résultats restant globalement groupés dans une même fourchette de pourcentage pour quatre des six animaux suivis ( 2 à $6 \%$ de formes $\mathrm{E}$ ). Ceci est confirmé par l'étude des moyennes par prélèvement (fig. $8 \mathrm{~A}$ ). Après 24 heures de séjour dans la solution hypoosmotique (fig. $8 \mathrm{~B}$ ), les pourcentages augmentent dans des proportions variables selon les animaux et la date 


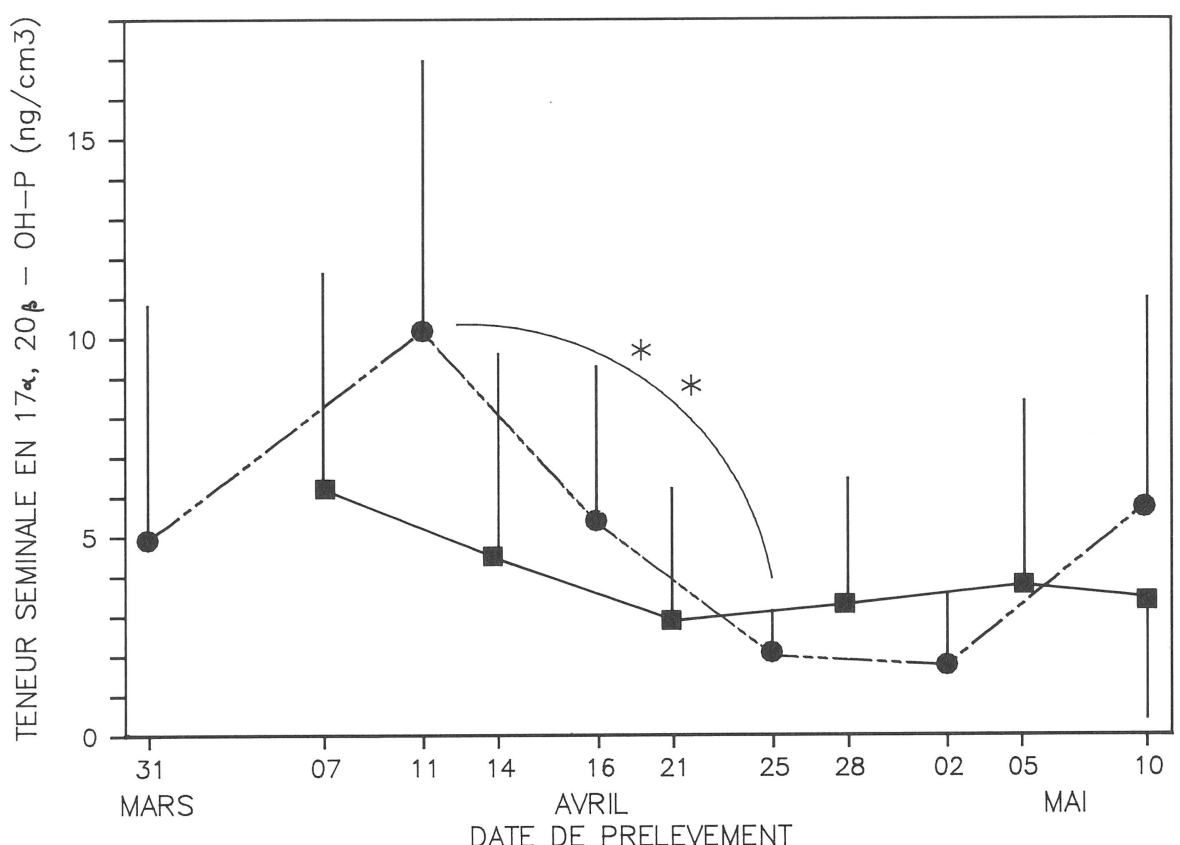

DATE DE PRELEVEMENT

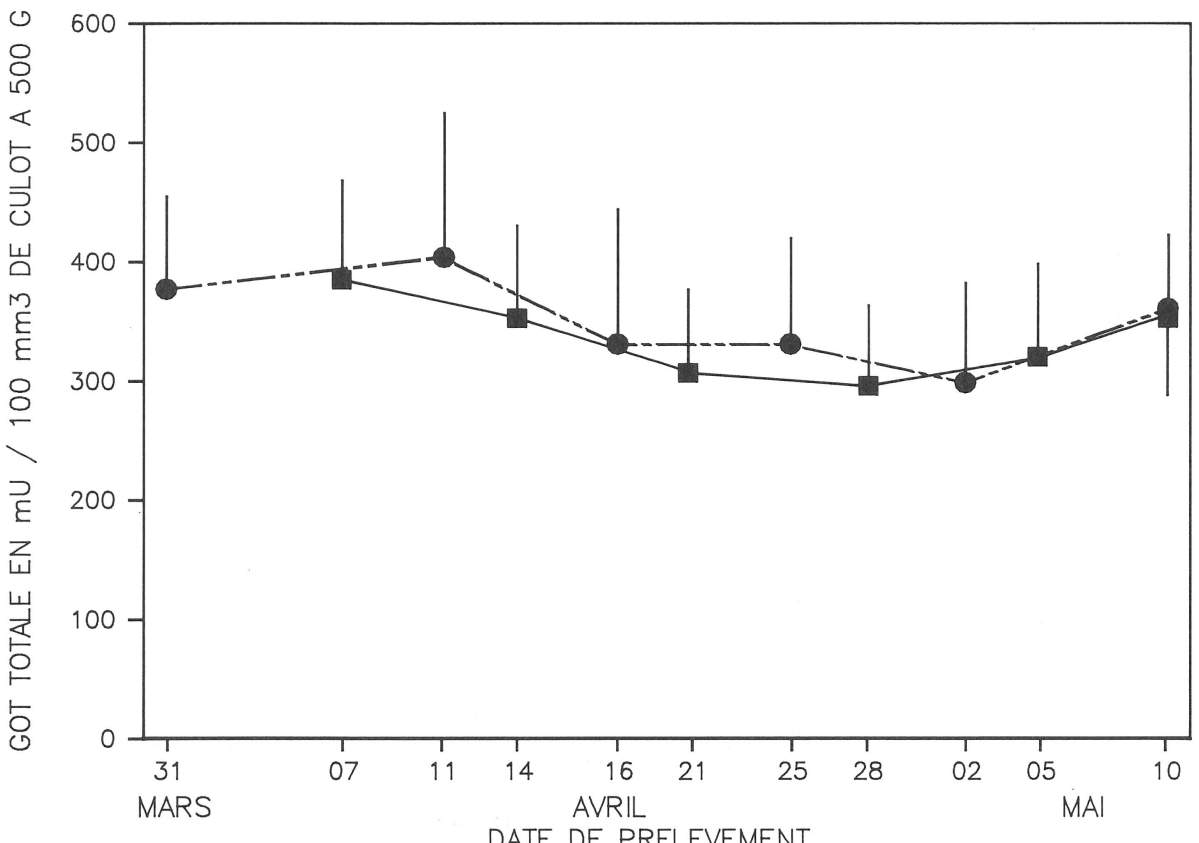

DATE DE PRELEVEMENT
Figure 6. - Évolution des moyennes des concentrations de $17 \alpha, 20 \beta-\mathrm{OH}-$ $\mathrm{P}\left(\mathrm{ng} / \mathrm{cm}^{3}\right.$ de plasma seminal) pour les deux lots de mâles (moyenne + écart type; $n=8$ à 10 spermes). Niveau de signification des différences observées (*) $p \leqq 0,05 ; \quad(* *)$ $p \leqq 0,01$.

Change in $17 \alpha, 20 \beta-O H-P \quad\left(\mathrm{ng} / \mathrm{cm}^{3}\right.$ seminal plasma) means for the two male group (mean $+S E ; n=8$ to 10 ). Significance level of observed difference $\left(^{*}\right) p \leqq 0.05 ;\left(^{* *}\right) p \leqq 0.01$.

Figure 7. - Évolution des moyennes de la concentration en glutamique oxaloacétique transaminase (GOT) totale contenue dans les spermatozoïdes $(\mathrm{mU} / 100 \mu \mathrm{l}$ de culot à $500 \mathrm{~g})$, pour les deux lots de mâles (moyenne + écart type; $n=8$ à 10 spermes). Niveau de signification des différences observées $\left({ }^{*}\right) p \leqq 0,05$; $\left.{ }^{* *}\right) p \leqq 0,01$.

Change in spermatozoa total content in glutamic oxaloacetic transaminase (GOT; $m U / 100 \mathrm{~mm}^{3} 500 \mathrm{~g}$ pellet) for the two male group (mean $+S E ; n=8$ to 10). Significance level of observed differences $\left({ }^{*}\right) p \leqq 0.05 ;\left({ }^{* *}\right) p \leqq 0.01$.

de prélèvement. On observe en effet une diminution significative au cours de la période de prélèvement, à partir du 18 avril. Dans les deux cas les évolutions sont semblables pour les deux lots.

L'évolution du pourcentage de spermatozoïdes non fluorescents est similaire pour les deux lots avec une baisse suivie d'une remontée significative fin avril, pour atteindre le niveau initial en fin d'expérimentation (fig. 9).

Pour l'analyse factorielle des correspondances, il n'a pas été tenu compte des formes de gonflement de types $\mathrm{G} 1$ et $\mathrm{G} 2$ en raison d'une possible subjectivité 

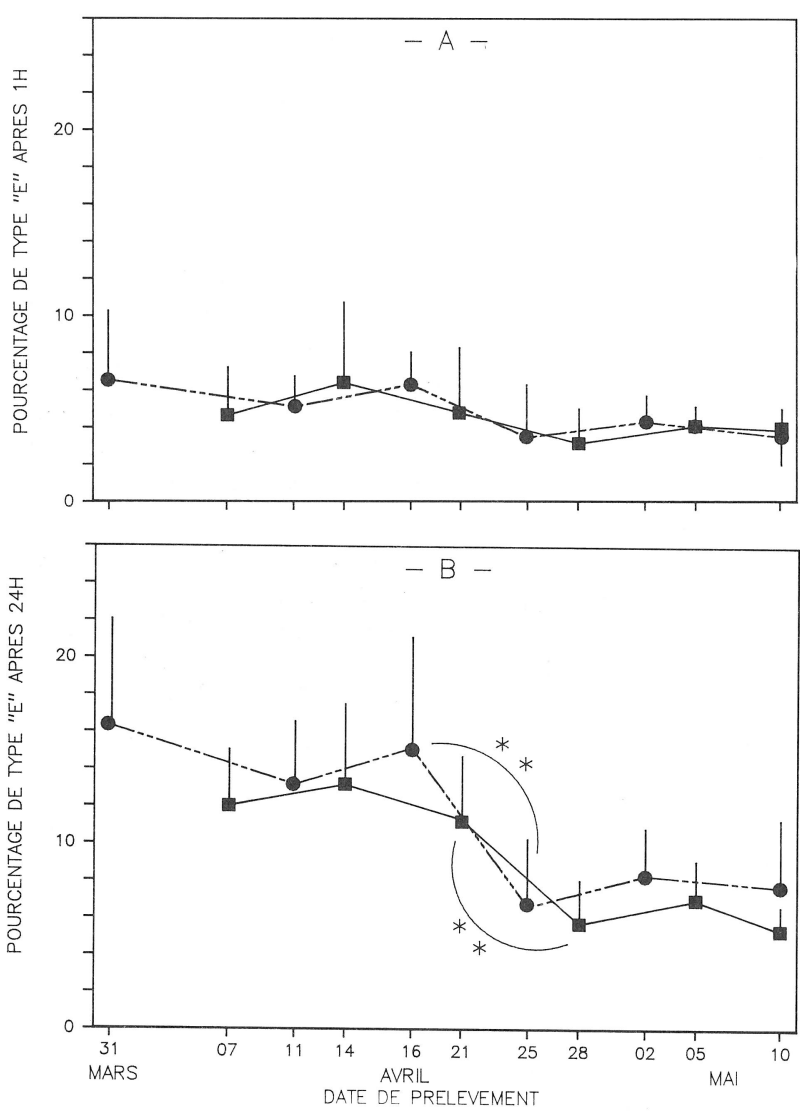

Figure 8. - Évolution des moyennes des pourcentages de spermatozoïdes de type $\mathbf{E}$ (membrane éclatée), après 1 (A) ou 24 heures (B) pour les deux lots de mâles (moyenne+écart type; $n=8$ à 10 spermes). Niveau de signification des différences observées $\left(^{*}\right)$ $p \leqq 0,05 ;(* *) p<0,01$.

Change in the percentage of $\mathbf{E}$ form (broken membrane) spermatozoa after 1 (A) or 24 hours (B) incubation, for the two male group (mean $+S E ; n=8$ to 10). Significance level of observed differences (*) $p \leqq 0.05 ;(* *) p \leqq 0.01$.

de ce paramètre. De même, les variables « concentration en spermatozoïdes », "niveau de $17 \alpha, 20 \beta-\mathrm{OH}-$ $\mathrm{P} »$, « GOT totale », « GOT plasma séminal frais » et « fécondance à l'état frais » n'ont pas été utilisées pour la représentation graphique en raison de leur absence évidente d'effet discriminatoire apparaissant à la lecture du tableau de contingence.

La représentation graphique des résultats de l'analyse factorielle des correspondances est donnée dans la figure 10. Les pourcentages d'inertie des axes 1, 2 et 3 sont respectivement de 75,0, 13,2 et 11,8\%.

Compte tenu des qualités de représentation de chaque point (nous avons retenu les modalités pour lesquelles la somme des cosinus carrés pour le plan est supérieure à 0,6), il apparaît que le plan 1-2 permet de suivre l'évolution des fécondances après congélation, des spermes médiocres (FEC1) aux très bons spermes (FEC4) en passant par ceux dits « moyens » $(\mathrm{FEC} 2)$ et « bons » $(\mathrm{FEC} 3)$.
Les spermes à fécondance médiocre (FEC1) sont associés à de forts pourcentages de GOT après décongélation ( $>40 \% ; \%$ GM 3 et $\%$ GM 4 ), à des pourcentages de formes de gonflement de type $\mathbf{E}$ supérieurs à $5 \%$ à 1 heure (e2) et supérieurs à $10 \%$ à 24 heures (E2) et à des pressions osmotiques séminales supérieures à 280 mOsm (PO3). Les meilleurs spermes sont associés à des pourcentages de GOT après décongélation inférieurs à $40 \%(\%$ GM 1 et $\%$ GM 2) à des pourcentages de forme de gonflement de type E, inférieurs à $5 \%$ à 1 heure (e1) et à $10 \%$ à 24 heures $(\mathrm{E} 1)$ et à des pressions osmotiques séminales inférieures à 280 mOsm (PO1 et PO2); parmi ceux-ci, les très bons spermes (FEC4) sont associés aux pourcentages les plus élevés de spermatozoïdes non fluorescents (NF4).

\section{DISCUSSION}

Les résultats des fécondations réalisées avec les spermes frais révèlent leur bonne qualité et confirme l'absence de mauvais spermes déjà observée à l'occasion d'autres expérimentations portant sur des collectes hebdomadaires (Maisse et al., 1988). Cependant une dilution à $10^{-4}$ aurait peut-être mis en évidence des différences entre spermes qui n'apparaissent pas à $10^{-3}$.

L'étude de la concentration en spermatozoïdes a fourni des valeurs supérieures à celles enregistrées par Büyükhatipoglu et Holtz (1984) et Maisse et al. (1988) dans les mêmes conditions. Le suivi individuel ainsi que l'évolution des moyennes par prélèvement a mis en évidence une tendance à la diminution des valeurs au cours de la période de spermiation, comme l'avaient déjà noté Büyükhatipoglu et Holtz (1984) et Munkittrick et Moccia (1987). Les écarts individuels enregistrés justifient a posteriori l'utilisation des culots de centrifugation à $500 \mathrm{~g}$ pour la congélation, de manière à homogénéiser le nombre de spermatozoïdes par boulette.

Les valeurs de l'osmolarité sont très dispersées comme cela a déjà été constaté par Maisse et al. (1988). La moyenne des résultats est proche de celle indiquée par Morisawa (1985), Erdahl (1986) et Maisse et al. (1988).

Les résultats concernant le dosage de la $17 \alpha, 20 \beta$ $\mathrm{OH}-\mathrm{P}$ libre sont originaux. En effet, bien que les spermatozoïdes soient capables de produire ce stéroïde (Ueda et al., 1984) qui par ailleurs est susceptible de contrôler la production de sperme (Baynes et Scott, 1985), il n'existe pas dans la littérature de données sur les variations de concentration de $17 \alpha$, $20 \beta-O H-P$ dans le plasma séminal de truite arc-enciel, mais seulement sur son niveau moyen (Baynes et Bye, 1987). Il aurait été intéressant de doser la $17 \alpha, 20 \beta-\mathrm{OH}-\mathrm{P}$ circulante pour savoir s'il existe une éventuelle relation avec la $17 \alpha, 20 \beta-\mathrm{OH}-\mathrm{P}$ séminale, mais les conditions expérimentales n'ont pas rendu 


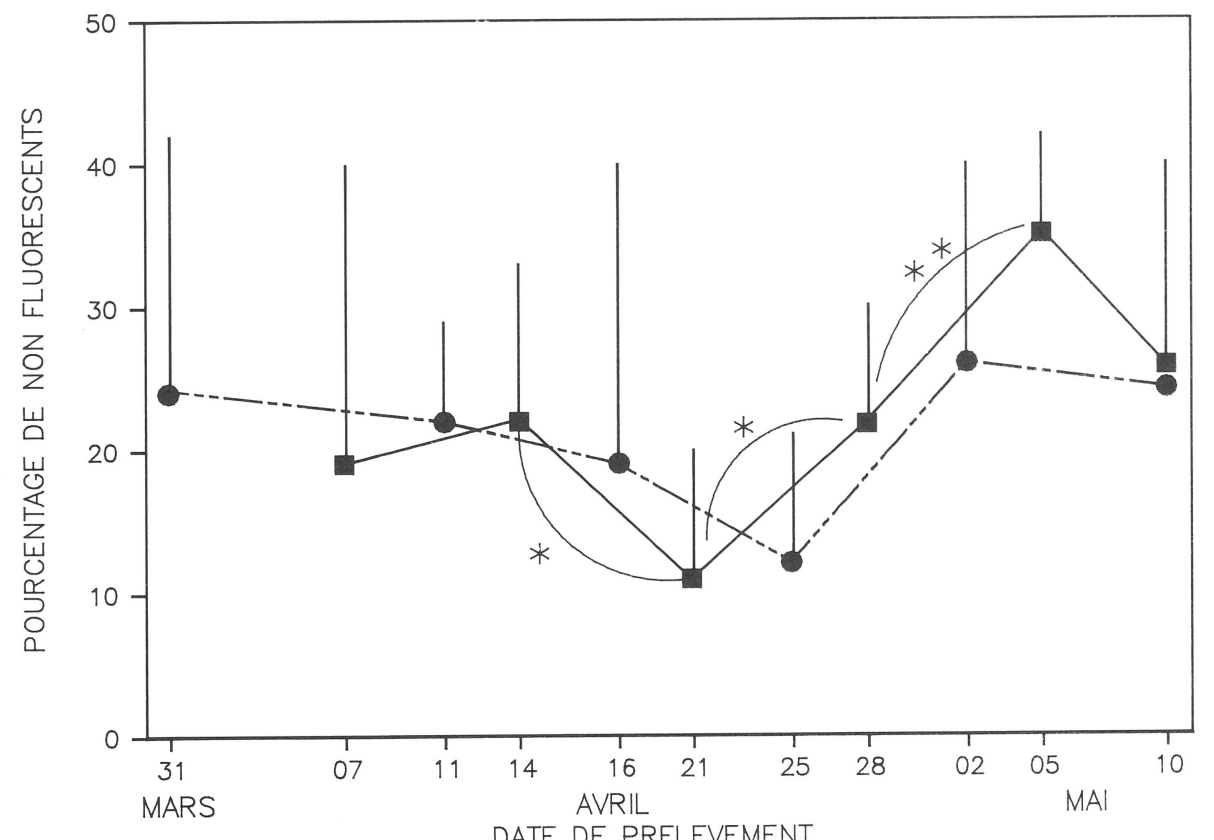

Figure 9. - Évolution des moyennes des pourcentages de spermatozoïdes non fluorescents (iodure de propidium) après incubation dans la solution à $20 \mathrm{mOsm}$ pour les deux lots de mâles, $\mathrm{A}$ et $\mathrm{B}$ (moyenne+écart type; $n=8$ à 10 spermes). Niveau de signification des différences observées $(*) p \leqq 0,05 ;(* *) p \leqq 0,01$.

Change in the percentage of non-fluorescent spermatozoa (propidium iodure) after incubation in a $20 \mathrm{mOsm}$ solution for the two male group (mean $+S E ; n=8$ to 10$)$. Significance level of observed difference (*) $p \leqq 0.05 ;(* *) p \leqq 0.01$. possible les prélèvements sanguins. Une étude réalisée chez 45 mâles spermiants d'une souche à reproduction automnale a mis en évidence l'absence de corrélation entre $17 \alpha, 20 \beta-\mathrm{OH}-\mathrm{P}$ plasmatique $\left(m \pm \mathrm{SE}=8,4 \pm 4,9 \mathrm{ng} / \mathrm{cm}^{3}\right)$ et $17 \alpha, 20 \beta-\mathrm{OH}-\mathrm{P}$ séminale $\left(m \pm \mathrm{SE}=2,2 \pm 2,0 \mathrm{ng} / \mathrm{cm}^{3}\right)$ (Loir, non publié). Enfin, il faut noter que la baisse enregistrée chez l'un des lots précède de peu la baisse de la pression osmotique séminale.

La concentration en GOT totale varie peu du début à la fin de l'expérience. Quant aux valeurs très faibles de GOT présente dans le plasma séminal, elles peuvent peut-être s'expliquer soit par le fait que lors de la dégradation des spermatozoïdes, cette enzyme mitochondriale ne serait libérée qu'au moment de la phase ultime, lorsque les mitochondries sont lésées, soit par une protéolyse rapide de l'enzyme.

Le dosage de la GOT libérée dans le dilueur après congélation-décongélation met en évidence l'effet protecteur du dilueur puisque les valeurs enregistrées sont comprises entre 21 et $59 \%$ de la GOT totale. Par ailleurs, des dosages effectués sur des boulettes homologues décongelées plus lentement ( 3 minutes au lieu de 10 secondes maximum) ont donné des valeurs proches de la GOT totale, confirmant que, dans nos conditions, l'essentiel des dommages subis par les membranes a lieu lors de la décongélation. En pratique, cette phase doit être la plus rapide possible, comme l'avaient déjà montré Schmidt et Holtz (1987) qui ont obtenu de meilleurs résultats pour une température de décongélation de $20^{\circ} \mathrm{C}$ au lieu de 5 et $10^{\circ} \mathrm{C}$.
Les différents types de réponse des spermatozoïdes à un choc hypoosmotique (formes G1, G2, E) observés chez la truite sont similaires à ceux précédemment décrits pour les spermatozoïdes humains (Jeyendran et al. 1984). Létude de la distribution des différents types à 0 et à 24 heures met en évidence l'évolution du type G1 (gonflement au niveau du flagelle) vers le type G2 (gonflement au niveau du flagelle et de la tête du spermatozoïde). Après 24 heures, le pourcentage de formes de type $\mathbf{E}$ augmente dans des proportions variables selon les spermes. Cette évolution reflète une perte progressive de l'intégrité membranaire pendant le séjour dans la solution hypotonique. Les formes $\mathbf{G} \mathbf{1}$ et $\mathbf{G} \mathbf{2}$ sont censées correspondre à des spermatozoïdes dont la membrane est intègre. Il en est de même pour les spermatozoïdes non fluorescents après incubation dans l'iodure de propidium. Les pourcentages observés pour ces derniers sont toujours inférieurs à ceux trouvés pour $\mathbf{G 1}+\mathbf{G} 2$. La concordance entre les évolutions des paramètres «Pression osmotique séminale» et «pourcentage de type $\mathbf{E}$ aprés 24 heures » semble indiquer que les spermatozoïdes résistent d'autant mieux au choc hypotonique que la pression osmotique séminale est basse : on ne sait pas si une pression osmotique élevée est la cause de la moindre résistance des spermatozoïdes ou si elle est la conséquence d'une fragilité membranaire intrinsèque (libération dans le plasma de molécules intra-cellulaires qui élèvent la pression osmotique). Cependant il n'existe pas de corrélation entre ces deux paramètres lorsque l'on considère l'ensemble des spermes pris individuellement. Ce paradoxe peut être 
expliqué par l'effet seuil imposé par l'osmolarité de la solution d'épreuve. Cet effet se retrouve fin avril, début mai, lorsque la réponse à la solution $60 \mathrm{mOsm}$ n'évolue plus alors que la résistance à la solution 20 mOsm augmente significativement.

La fécondance du sperme congelé montre une large distribution déjà mise en évidence lors d'une étude précédente sur la truite arc-en-ciel (Maisse et al., 1988) bien qu'une différence essentielle entre les deux expériences ait été apportée lors de la réalisation des boulettes : dans notre cas, c'est le culot débarrassé du plasma séminal qui a été dilué puis congelé et non le sperme total. L'augmentation des fécondances notée pour l'un des lots à la fin de la période d'expérimentation a déjà été observée (Schmidt, 1985) en fin de spermiation. Sur une durée d'expérimentation double (12 semaines) Maisse et al. (1988) avaient observé deux périodes d'augmentation de la fécondance après congélation suivies d'une diminution importante (résultats non publiés). En l'état actuel de nos connaissances, ces diverses observations sont difficilement interprétables par manque d'information sur le vieillissement intratesticulaire du sperme.

L'analyse des performances de chaque sperme congelé en relation avec les paramètres étudiés confirme l'hypothèse de la relation « qualité membranaire des spermatozoïdes/fécondance après cryoconservation 》 formulée par Baynes et Scott (1982), Yoo et al. (1987) et Maisse et al. (1988). En effet ce sont les spermatozoïdes dont la membrane résiste le mieux au choc hypoosmotique (faible pourcentage de formes de type $\mathbf{E}$ à 1 heure comme à 24 heures ou forts pourcentages de noyaux non fluorescents) qui supportent le mieux la congélation. A ces spermes sont aussi associées des faibles concentrations de GOT retrouvée après la décongélation $(<40 \%$ de la quantité totale), ce qui indique une meilleure résistance de la membrane. Cependant, l'absence de corrélation forte entre les paramètres membranaires et l'aptitude à la congélation nous amène à envisager parallèlement au rôle de la membrane des spermatozoïdes, l'influence éventuelle de facteurs du plasma séminal,

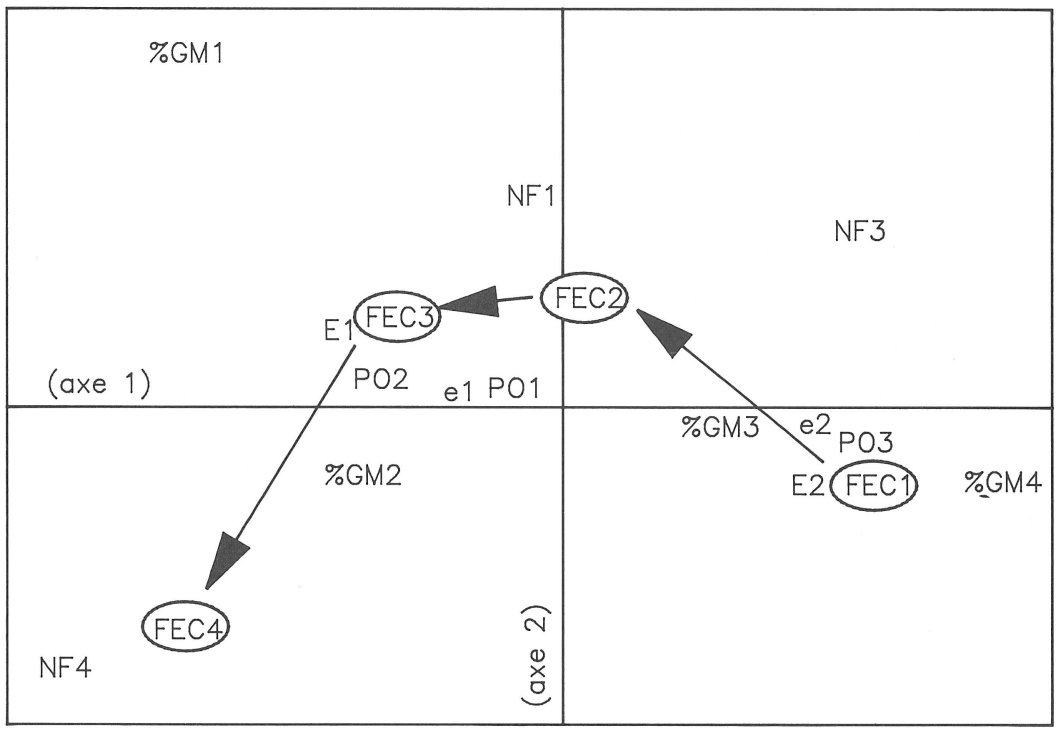

Figure 10. - Relations entre la fécondance du sperme congelé et les caractéristiques physico-chimiques et les résultats des différents tests de qualité membranaire dans le plan des deux premiers axes factoriels issus de l'analyse du tableau de contingence. Seules les modalités dont la somme des $\operatorname{Cos}^{2}$ est pour le plan supérieur à 0,6 sont représentées :

- formes de gonflement de type $\mathbf{E}$ à 1 heure : e1 $(1,3<p<5 \%)$ et e2 $(5<p<30 \%)$;

- formes de gonflement de type E à 24 heures : E1 $(3,6<p<10 \%)$ et E2 $(10<p<30 \%)$;

- spermatozoïdes non fluorescents : NF2 $(10<p<20 \%)$, NF3 $(20<p<30 \%)$ et NF4 $(30<p<80 \%)$;

- pression osmotique séminale : PO1 (PO $<250 \mathrm{mOsm}), \mathrm{PO} 2(250<\mathrm{PO}<280 \mathrm{mOsm})$ et $\mathrm{PO} 3(\mathrm{PO}>280 \mathrm{mOsm})$;

- GOT dans le dilueur de congélation (exprimé en pourcentage de la GOT totale) : \% GM1 ( $p<30 \%)$, $\%$ GM2 $(30<p<40 \%)$, $\%$ GM3 $(40<p<50 \%), \%$ GM4 $(p>50 \%)$.

Relationship of frozen milt fertility versus physico-chemical characteristics and membrane quality: the first two factorial axes from analysis of the table of contingence. Only modalities with a $\mathrm{Cos}^{2}$ sum higher than 0.6 for the plane are shown:

- E forms at 1 hour: e1 $(1.3<p<5 \%)$ and $e 2(5<p<30 \%)$;

- E forms at 24 hours: E1 $(3.6<p<10 \%)$ and $E 2(40<p<60 \%)$

- Non-fluorescent spermatozoa: NF2 $(10<p<20 \%), N F 3(20<p<30 \%)$, NF4 $(30<p<80 \%)$;

- Seminal osmolality: PO1 (PO<250 mOsm), PO2 $(250<P O<280 \mathrm{mOsm})$ and PO3 (PO $>280 \mathrm{mOsm})$;

- GOT in the diluent (in percent of the GOT total level): \% GM1 $(p<30), \%$ GM2 $(30<p<40), \% G M 3(40<p<50), \%$ GM4 $(p>50)$. 
liés à la physiologie du mâle et pouvant être liés au fonctionnement de la barrière "sang-testicule". Ainsi, par exemple, cette dernière qui a été localisée entre le sang et les spermatozoïdes chez Aphanius dispar par Abraham et al. (1980), jouerait un rôle dans la sécrétion de l'ion potassium indispensable au maintien de l'immobilité des spermatozoïdes (Marshall et Bryson, 1988) mais aussi peut-être dans la sécrétion d'autres ions et/ou molécules. En effet, la relation mise en évidence par Maisse et al. (1988) entre les fortes pressions osmotiques séminales et l'inaptitude des spermes à la congélation-décongélation a été retrouvée dans nos conditions où la plus grande partie du plasma séminal avait été ôtée avant congélation.

\section{CONCLUSION}

Cette étude nous a permis de démontrer l'existence d'une qualité membranaire des spermatozoïdes varia- ble d'un éjaculat à l'autre et influençant l'aptitude à la congélation du sperme.

Par ailleurs, d'un point de vue pratique, nous pouvons dès maintenant proposer deux techniques simples pour évaluer la qualité d'un éjaculat :

$1^{\circ}$ la mesure de la pression osmotique séminale, conduisant au rejet de ceux pour lesquels elle est supérieure à $280 \mathrm{mOsm}$;

$2^{\circ}$ l'évaluation de la qualité de la membrane par la mise en évidence de sa résistance à un séjour prolongé ( 24 heures) dans une solution hypotonique (60 mOsm) : les spermes pour lesquels le pourcentage de spermatozoïdes dont la membrane a éclaté est supérieur à $10 \%$ étant éliminés.

Ces deux techniques restent cependant imparfaites et, en laboratoire, la quantité de GOT dans le dilueur, après décongélation rapide, rapportée à celle de GOT « totale » nous paraît un meilleur paramètre à considérer, en absence de possibilité de test direct de la fécondance.

\section{Remerciement}

Ce travail a été financé partiellement par un contrat de l'Institut National de la Recherche Agronomique (GCS-BBA, 1989).

\section{RÉFÉRENCES}

Abraham M., E. Rahamin, H. Tibika, E. Golenser, M. Kielselstein, 1980. The blood testis barrier in Aphanius dispar (Teleostei). Cell. Tissue Res., 211, 207-214.

Baynes S. M., V. J. Bye, 1987. Seminal fluid production in rainbow trout (Salmo gairdneri). Proc. 3rd Int. Symp. on Reproduction Physiology of Fish, St John's, Canada, 247.

Baynes S. M., A. P. Scott, 1982. Cryopreservation of rainbow trout spermatozoa: variation in membrane composition may influence spermatozoan survival. In: Reproductive physiology of fish. Richter C. J. J., H. S. Th. Goos ed., PUDOC, Wageningen, 128.

- 1985. Seasonal variations in parameters of milt production and in plasma concentration of sex steroids of male rainbow trout (Salmo gairdneri). Gen. Comp. Endocrinol., 57, 150-160.

Benzecri J. P., 1980. L'analyse de données; tome 1 : La taxinomie; tome 2: L'analyse des correspondances, Dunod, Paris, 2 vol., 625 et 632 p.

Billard R., 1977. Utilisation d'un système tri-glycocolle pour tamponner le dilueur d'insémination pour truite. Bull. Fr. Pisc., 264, 102-112.

Billard R., B. Jalabert, 1974. L'insémination artificielle de la truite (Salmo gairdneri, Richardson). II. Comparaison des effets des différents dilueurs sur la conservation de la fertilité des gamètes avant et après insémination. Ann. Biol. anim. Bioch. Biophys., 14, 601-610.
Büyükhatipoglu S., W. Holtz, 1984. Sperm output in rainbow trout (Salmo gairdneri). Effect of age, timing and frequency of stripping and presence of females. Aquaculture, 37, 63-71.

Erdhal D. A., 1986. Preservation of spermatozoa and ova from freshwater fish. Ph. D Univ. Thesis, Minnesota, $308 \mathrm{p}$.

Fostier A., B. Jalabert, 1986. Steroidogenesis in Rainbow trout at various preovulatory stages: changes in plasma hormone levels and in vivo and in vitro responses of the ovary to Salmon gonadotropin. Fish Physiol. Biochem., 2, 87-89.

Foucart T., 1984. Analyse factorielle - Programmation sur micro-ordinateurs et Analyse factorielle de tableaux multiples. Masson, 234 et 185 p.

Jeyendran, R. S., H. H. Van Der Ven, M. Perez-Pelaez, B. G. Crabo, L. J. D. Zaneveld, 1984. Development of an assay to assess the functional integrity of the human sperm membrane and its relationship to other semen characteristics. J. Report. Fert., 70, 219-228.

Legendre M., R. Billard, 1980. Cryoconservation du sperme de truite arc-en-ciel (Salmo gairdneri R.). Bull. Fr. Pisc., 278, 11-33.

Maisse G., A. Pinson, M. Loir, 1988. Caractérisation de l'aptitude à la congélation du sperme de truite arc-enciel (Salmo gairdneri) par des critères physico-chimiques. Aquat. Living Resour., 1, 45-51.

Marshall W. S., S. E. Bryson, 1987. Evidence for $\mathrm{Cl}^{-}$dependent $\mathrm{K}^{+}$secretion by the blood-testis barrier of brook trout. Can. J. Zool., 66, 1603-1609. 
Morisawa M., 1985. Initiation mechanism of sperm motility at spawning in teleosts. Zool. Sci., 2, 605-615.

Munkittrick K. R., R. D. Moccia, 1987. Seasonal changes in the quality of rainbow trout (Salmo gairdneri) semen: effect of a delay in stripping on spermatocrit, motility, volume and seminal plasma constituents. Aquaculture, 64, 147-156.

Nagase H., 1964. Deep freezing bull semen in concentrated pellet form. I. Factors affecting survival of spermatozoa. Proc. Int. Congr. Reprod. Anim. Insem. Artif., Trento, 4, 410-415.

Schmehl M., E. F. Graham, D. A. Erdahl, 1987. Chemical constituents of trout seminal plasma after minimal and maximal cell damage treatments with possible applications to semen evaluations assays. Aquaculture, 62, 311318.

Schmidt R., 1985. Untersuchungen zur gefrierkonservierung und gefriereignung von regenbogen-forellensperma. Dokdissertation, Göttingen.

Schmidt R., W. Holtz, 1987. Effect of pellet size and thawing temperature on fertility of frozen-thawed Rainbow trout (Salmo gairdneri) sperm. Proc. 3rd Int. Symp. on Reproductive Physiology of Fish, St John's, Canada, 119.

Scott A. P., S. M. Baynes, 1980. A review of the biology, handling and storage of salmonid spermatozoa. J. Fish Biol., 17, 707-739.

Stoss J., 1983. Fish gamete preservation and spermatozoan physiology. In: Fish Physiology, vol. IX, Reproduction, part B., behavior and fertility control. Hoar W. S., Randall D. J., Donaldson E. M. ed., Academic Press, New York. 305-350.

Stoss J., W. Holtz, 1983. Cryopreservation of rainbow trout (Salmo gairdneri) sperm. III. Effect of proteins in the diluent sperm from different males and interval between sperm collection and freezing. Aquaculture, 31, 275-282.

Ueda H., A. Kambegawa, Y. Nagahama, 1984. In vitro 11Ketotestosterone and $17 \propto 20 \beta$-dihydroxy-4-pregen-3-one production by testicular fragments and isolated sperm of Rainbow Trout. J. Exp. Zool., 231, 435-439.

Yoo B. Y., M. A. Ryan, A. J. Wiggs, 1987. Loss of protein from spermatozoa of atlantic salmon (Salmo salar L.) because of cryopreservation. Can. J. Zool., 65, 9-13. 